\title{
RTWPMS: A Real-Time Wireless Physiological Monitoring System
}

\author{
Bor-Shing Lin, Bor-Shyh Lin, Member, IEEE, Nai-Kuan Chou, Fok-Ching Chong, and Sao-Jie Chen
}

\begin{abstract}
This paper demonstrates the design and implementation of a real-time wireless physiological monitoring system for nursing centers, whose function is to monitor online the physiological status of aged patients via wireless communication channel and wired local area network. The collected data, such as body temperature, blood pressure, and heart rate, can then be stored in the computer of a network management center to facilitate the medical staff in a nursing center to monitor in real time or analyze in batch mode the physiological changes of the patients under observation. Our proposed system is bidirectional, has low power consumption, is cost effective, is modular designed, has the capability of operating independently, and can be used to improve the service quality and reduce the workload of the staff in a nursing center.
\end{abstract}

Index Terms-Nursing center, real-time wireless physiological monitoring system (RTWPMS), wireless communication.

\section{INTRODUCTION}

$\mathbf{P}$ ROGRESS of medical technologies and development of newer and better medicines have resulted in a rapid increase of the aged population. Thus, the requirements of rest homes and rehabilitation centers increase day by day. Also, more medical personnel are needed to offer medical treatments and to prevent accidents to aged patients. To provide these aged patients with a more humane environment for their physical and physiological heath care, frequent monitoring and recording of their physiological status becomes very important [1]-[6]. The number of staff in a nursing center assigned to regularly observe and record the physiological status of patients occupies a large portion of that center's human resources. Even if we have sufficient professional nursing staff who works very cautiously and conscientiously, we still cannot guarantee to obtain the necessary patient status information on time and to prevent accidents from happening.

To reduce the workload of the nursing staff and prevent sudden situations that cause accidents, many measurements have been proposed. Firstly, we have to design a physiological status examination device for the staff to collect the physiological

Manuscript received November 15, 2004; revised April 14, 2005 and November 12, 2005. This work was supported by the National Science Council, Taiwan, R.O.C., under Grant NSC-93-2215-E002-032.

B.-S. Lin, B.-S. Lin, and F.-C. Chong are with the Department of Electrical Engineering, National Taiwan University, Taipei 106, Taiwan, R.O.C. (e-mail: d88921027@ntu.edu.tw; d88921033@ntu.edu.tw; cchong@cc.ee.ntu.edu.tw).

N.-K. Chou is with National Taiwan University Hospital, Taipei 100, Taiwan, R.O.C. (e-mail: nickchou@ha.mc.ntu.edu.tw).

S.-J. Chen is with the Department of Electrical Engineering and Graduate Institute of Electronics Engineering, National Taiwan University, Taipei 106, Taiwan, R.O.C. (e-mail: csj@cc.ee.ntu.edu.tw).

Digital Object Identifier 10.1109/TITB.2006.874194 status information from patients in real time. After the information collection, the monitoring device should be responsible for transmitting the information to the nursing staff via any of the following medium: Wired data transmission, public service network [7], microwave [8], public [9]-[13] or private [14]-[16] wireless network, and the Internet [17]. Some popular wireless communication techniques in telemedicine are compared in Table I. The concepts of physiological monitoring and telemedicine can thus be used to relieve or even replace parts of the staff loading.

Historically, a physiological monitoring system is mainly either a real-time personal monitoring device or a remote home care device with batch-mode information processing. These systems usually do not consider the aspects of mobile, multiuser, real-time, low-power, low-cost, and bidirectional communication. Because of these drawbacks, we propose a real-time wireless physiological monitoring system (RTWPMS) for nursing centers, which comprises mobile physiological examination device(s), wireless base station(s), a voice/data exchange device, and a network management center. A modular design approach is used to develop both the hardware and software of the RTWPMS. The mobile physiological examination devices, with mobile, low-power, low-cost, and modular design features, are used to collect patient vital signals and then transmit real-time data to a network management center. The wireless base stations allow the communication or hand-off between mobile physiological examination devices. The voice/data exchange device is used to provide voice service to the medical staff in a nursing center and to transmit the data collected from mobile wireless medical devices to the network management center. The function of a network management center is to monitor the status of the patients and to provide data storage and analysis.

\section{System ARChiteCture AND DeSIGN}

The construction of an RTWPMS is based on a wireless communication network; the system architecture is shown in Fig. 1. The RTWPMS hardware consists mainly of four major components: mobile physiological examination device(s); wireless base station(s); a voice/data exchange device; and a network management center. These hardware components combined with human-machine interface software form a real-time physiological monitoring system.

\section{A. Mobile Physiological Examination Device}

The patient-wearing mobile physiological examination device mainly consists of a wireless communication module and a built-in medical examination module, as shown in Fig. 2. The 
TABLE I

COMPARISON OF WIRELESS COMMUNICATION TECHNIQUES IN TELEMEDICINE

\begin{tabular}{lcccccc}
\hline & GSM & GPRS & PHS & $\begin{array}{c}\text { WLAN } \\
(802.11 \mathrm{~b})\end{array}$ & Microwave & $\begin{array}{c}\text { CT2 } \\
\text { (Used in RTWPMS) }\end{array}$ \\
\hline Transfer Rate & $9.6 \mathrm{~kb} / \mathrm{s}$ & $115 \mathrm{~kb} / \mathrm{s}$ & $64 \mathrm{~kb} / \mathrm{s}$ & $11 \mathrm{Mb} / \mathrm{s}$ & $1 \sim 10 \mathrm{Mb} / \mathrm{s}$ & $32 \mathrm{~kb} / \mathrm{s}$ \\
\hline Bi-directional Communication & Yes & Yes & Yes & Yes & Yes & Yes \\
\hline Use in the Hospital & No & No & Yes & Yes & No & Yes \\
\hline Range & Large & Large & Large & Small & Large & Selective \\
\hline Cost & High & High & High & Medium & High & Low \\
\hline Modular Design & No & No & No & No & No & Yes \\
\hline Operating Independently & No & No & No & Yes & No & Yes \\
\hline
\end{tabular}

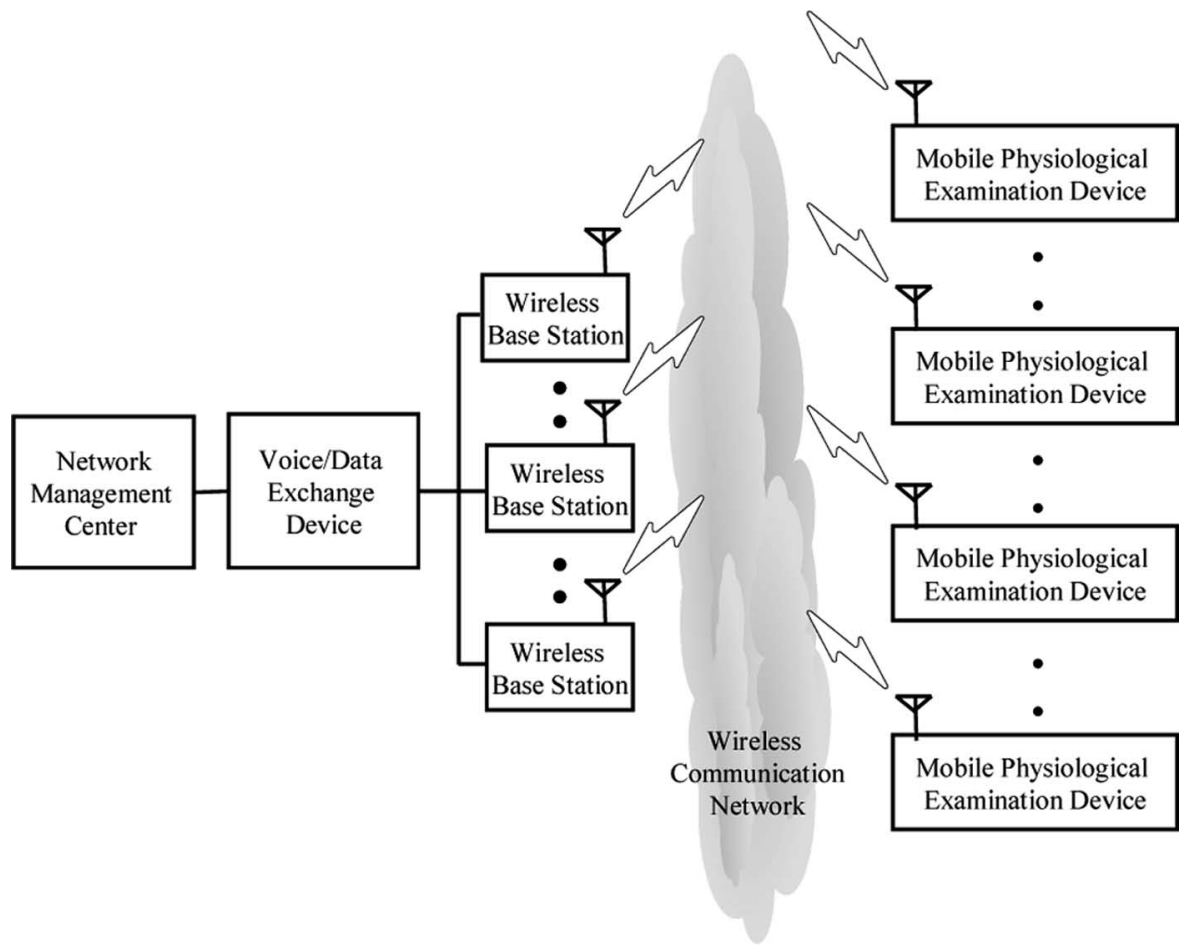

Fig. 1. System architecture of an RTWPMS.

wireless communication module is a digital and low-power second generation cordless telephone (CT2) module (TRF-RE202, SONY Corporation, Korea), operated in the 864-MHz industrial, scientific, and medical (ISM) band. The built-in medical examination module is used to receive commands from the wireless communication module and to check patient physiological information periodically. This device also provides parameters setting, patient identification number setting, warning setting, and working mode setting. The staff of a nursing center can then use this identification number to identify or locate a patient.

This device can be used to transmit voice and data information. For voice transmission, analog signals have to be converted into digital signals first. Since the voice bandwidth is $4 \mathrm{kHz}$, the hardware uses a sampling rate of $8 \mathrm{kHz}$ to sample signals and prevent signal aliasing. As to the voice and data coding, we first use 8-b pulse code modulation (PCM) to modulate and generate data with a rate of $64 \mathrm{~kb} / \mathrm{s}$. Then, adaptive differential PCM (AD$\mathrm{PCM})$ is used for voice and data compression to reach a transfer rate less than $32 \mathrm{~kb} / \mathrm{s}$. Blood pressure (BP), heart rate (HR), and temperature measured by the built-in medical examination module are transmitted as data. Temperature and HR sensors can measure vital signals in real time and transfer them through the data transmission interface. BP measured from a cuff is set to be measured per hour. If the patient feels uncomfortable with automatic measurements, then he has the option to manually start the BP measurement. When necessary, we can add on medical examination modules, such as electrocardiogram (ECG), oxyhemoglobin saturation by pulse oximetry $\left(\mathrm{SpO}_{2}\right)$, to the device. This device also provides a data transmission interface to link to an external global positioning system (GPS) module. The working mode of this data transmission interface can be remotely set by the medical staff of a network management center via the wireless communication network. Equipped with a GPS module, patients having activities outdoors can also be tracked by the staff of a network management center. The data transmission interface provides four RS-232 ports to link with at most four different medical examination modules. Each RS-232 has a maximal 19.2-kb/s transfer rate and provides enough speed to most medical examination modules and bedside monitors. The RS-232 port has adjustable communication parameters set by 


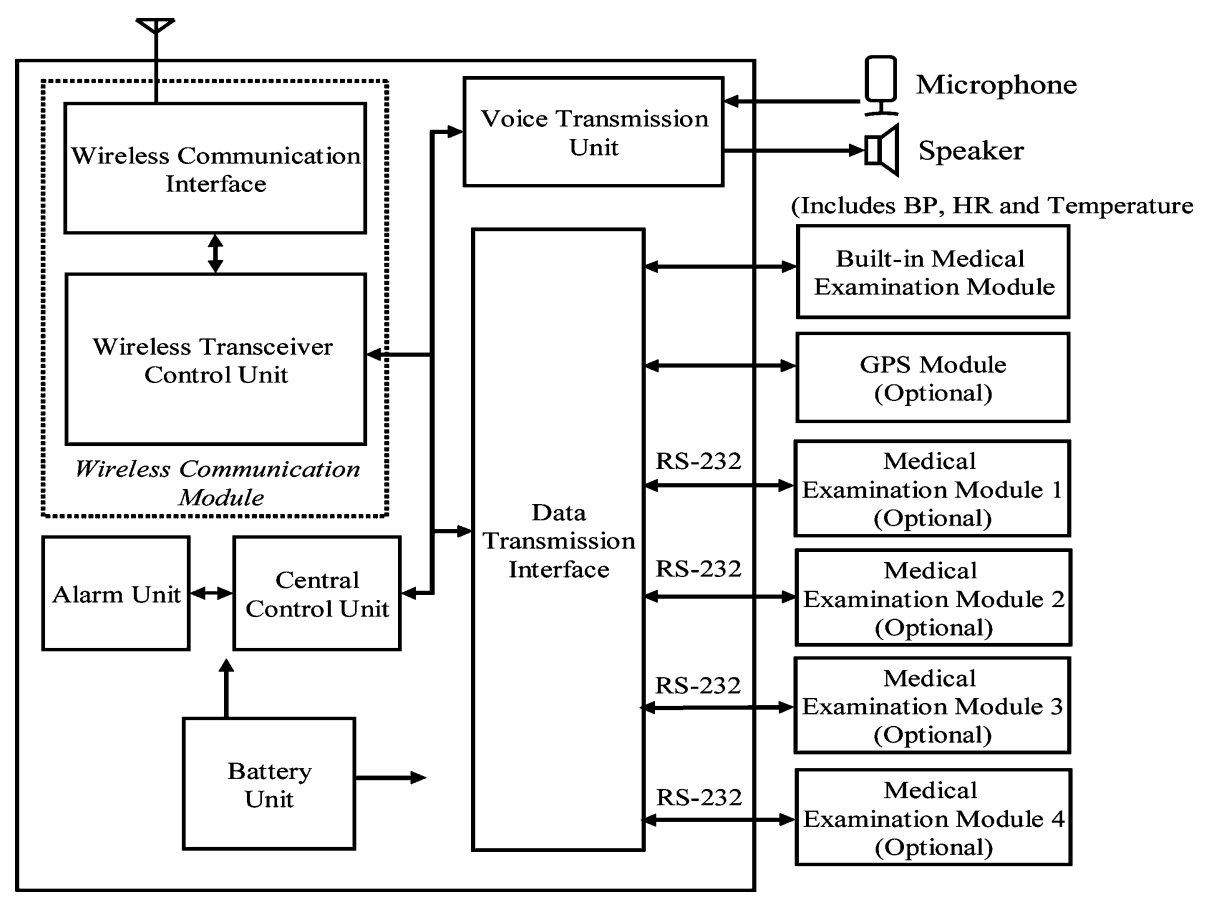

Fig. 2. Block diagram of a mobile physiological examination device.

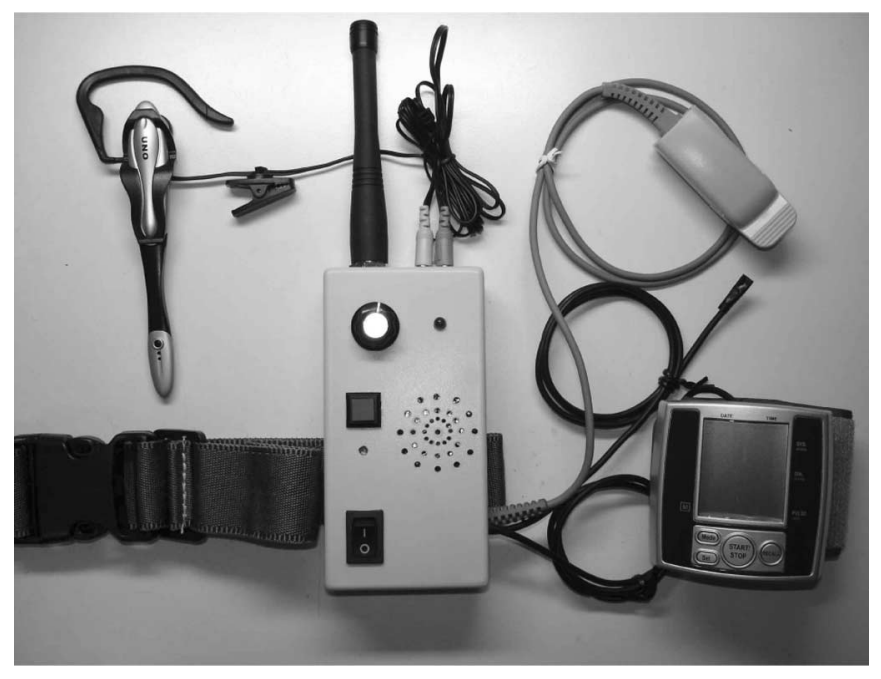

Fig. 3. Photograph of the mobile physiological examination device.

software and the total transfer rate on this interface is $32 \mathrm{~kb} / \mathrm{s}$. If the data transmission interface needs higher speed, RS-232 can be upgraded to universal serial bus (USB). When these data transmission ports are activated, we must process the total data flow control with a central control unit, which uses a softwarecontrolled memory as the transmission buffer. In sending or receiving information, we use digital compression, encryption, and identification processes to improve the accuracy and security of data transmitted in a wireless channel. Fig. 3 illustrates the mobile physiological examination device with three built-in medical examination modules. Its size and weight are suitable for aged patients.

\section{B. Wireless Base Station}

The main function of a wireless base station is to send and receive radio wave data and commands from/to the mobile physiological examination device and voice/data exchange device. It consists of a wireless communication interface, a wireless base station control unit, a data transmission interface, and a power supply circuit, as shown in Fig. 4. The system is controlled by software installed in the wireless base station control unit. A wireless base station uses digital modulation signals to communicate with the voice/data exchange device. The data are encoded with a nonreturn-to-zero (NRZ) coding. NRZ is a binary encoding scheme in which ones and zeroes are represented by opposite and alternating high and low voltages and where there is no return to a zero voltage between encoded bits. That is, the stream has only two values, low and high. The NRZ coding keeps signal status until the signal level is from high to low or low to high. Furthermore, the NRZ has the following advantages: low cost; stablility; and easy implementation. Transmitting modulated digital signals helps to increase the distance between a voice/data exchange device and wireless base stations, thus reducing the probability of error.

\section{Voice/Data Exchange Device}

The major function of a voice/data exchange device is to exchange, receive, and process voice and data messages. The device receives control commands from a network management center to establish communication and control with wireless base stations. As shown in Fig. 5, the voice/data exchange device consists of the following components: an analog voice interface; a voice/data exchange control unit; a data transmission 


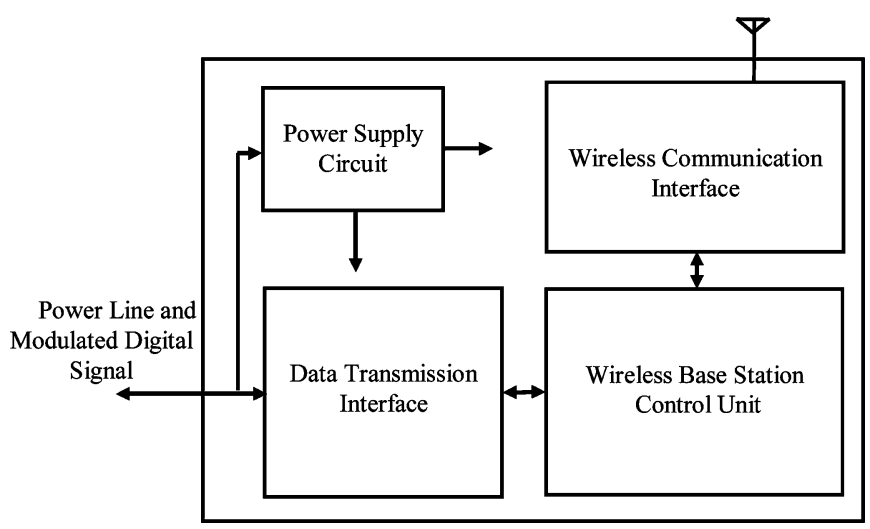

Fig. 4. Block diagram of a wireless base station device.

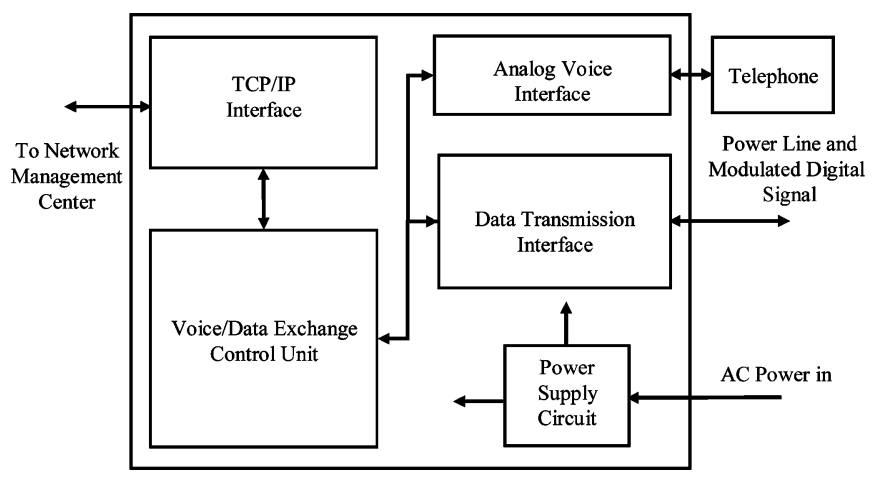

Fig. 5. Block diagram of a voice/data exchange device.

interface linked to the wireless base station; a TCP/IP interface linked to the network management center; and a power supply circuit. Related application programs are installed in the voice/data exchange control unit.

In the data transmission interface, we use the modulated digital signals to communicate with wireless base stations and we use the NRZ code in these signals to increase the distance of data transmission and reduce the probability of error.

This data transmission interface provides digital modulation signals and carries a $-48-\mathrm{V}$ dc power supply required by wireless base stations. In our design, the RTWPMS needs only one voice/data exchange device, which can supply power to a maximal of 60 base stations. We cable every base station separately with a 9-mm cable line and at a maximal distance of $1 \mathrm{~km}$ from the voice/data exchange device. The modulated digital signals use an alternate mark inversion (AMI) signal code such that the $-48-\mathrm{V}$ dc power supply provided by the voice/data exchange device can carry the modulated digital signals. For communication between the voice/data exchange device and the network management center, the voice/data exchange device uses a highspeed TCP/IP network to transmit the mass of data collected from wireless base stations.

\section{Network Management Center}

The network management center is the center of data processing and status control. Through this center, the nursing staff can control the voice/data exchange device, wireless base sta-

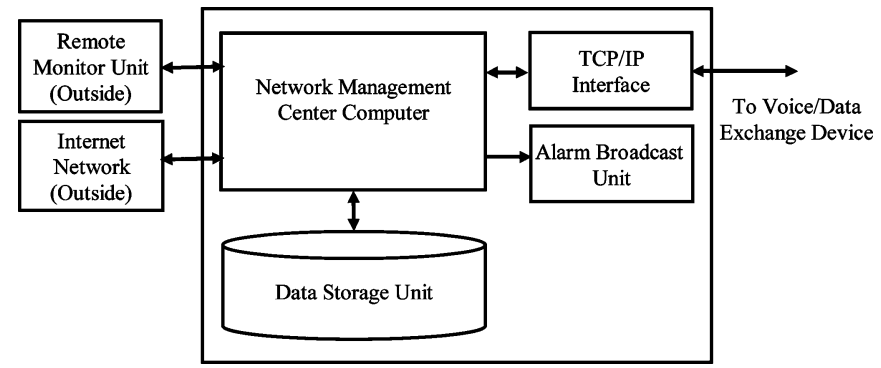

Fig. 6. Block diagram of a network management center.

tions, and mobile physiological examination devices to monitor the vital signals of aged patients. As shown in Fig. 6, the network management center is composed of a network management center computer, a data storage unit, a TCP/IP interface, and an alarm broadcast unit. The computer can connect to different hospitals through the Internet.

\section{SYSTEM SOFTWARE DESIGN}

The software of our system has been designed based on a modular approach. The overall software architecture of our RTWPMS is shown in Fig. 7. Four sets of software application programs have been developed, respectively, for mobile physiological examination device, wireless base station, voice/data exchange device, and network management center. Each set of application programs is implemented in a modularized tree-like hierarchical structure using an object-oriented method. Thus, all the programs developed are easy to update, modify, and maintain. The detailed design of each set of application programs is described as follows.

\section{A. Software in Communication Equipments}

The set of application programs installed in the mobile physiological examination device mainly includes modules for physiological signal examination, voice processing, data transmission control, wireless communication control, and status processing. Since the application program was designed on the basis of a tree-like hierarchical structure that centralizes all common functions to have a more efficient execution, we can rapidly execute any function according to the input status and requirement in that module after system initialization. Under this structure, we can easily add a new function into a module or remove another from a module.

The physiological signal examination module includes functions of measuring body temperature, BP, and HR. The setup of each examination device's parameters and measuring cycle is performed in this software module.

The application program in mobile physiological examination device also manages voice processing, data transmission, wireless communication, and status processing. Before voice and data are transmitted, this program compresses and processes those data. The mobile physiological examination device can, thus, reduce the amount of data transmitted and increase the channel bandwidth. 


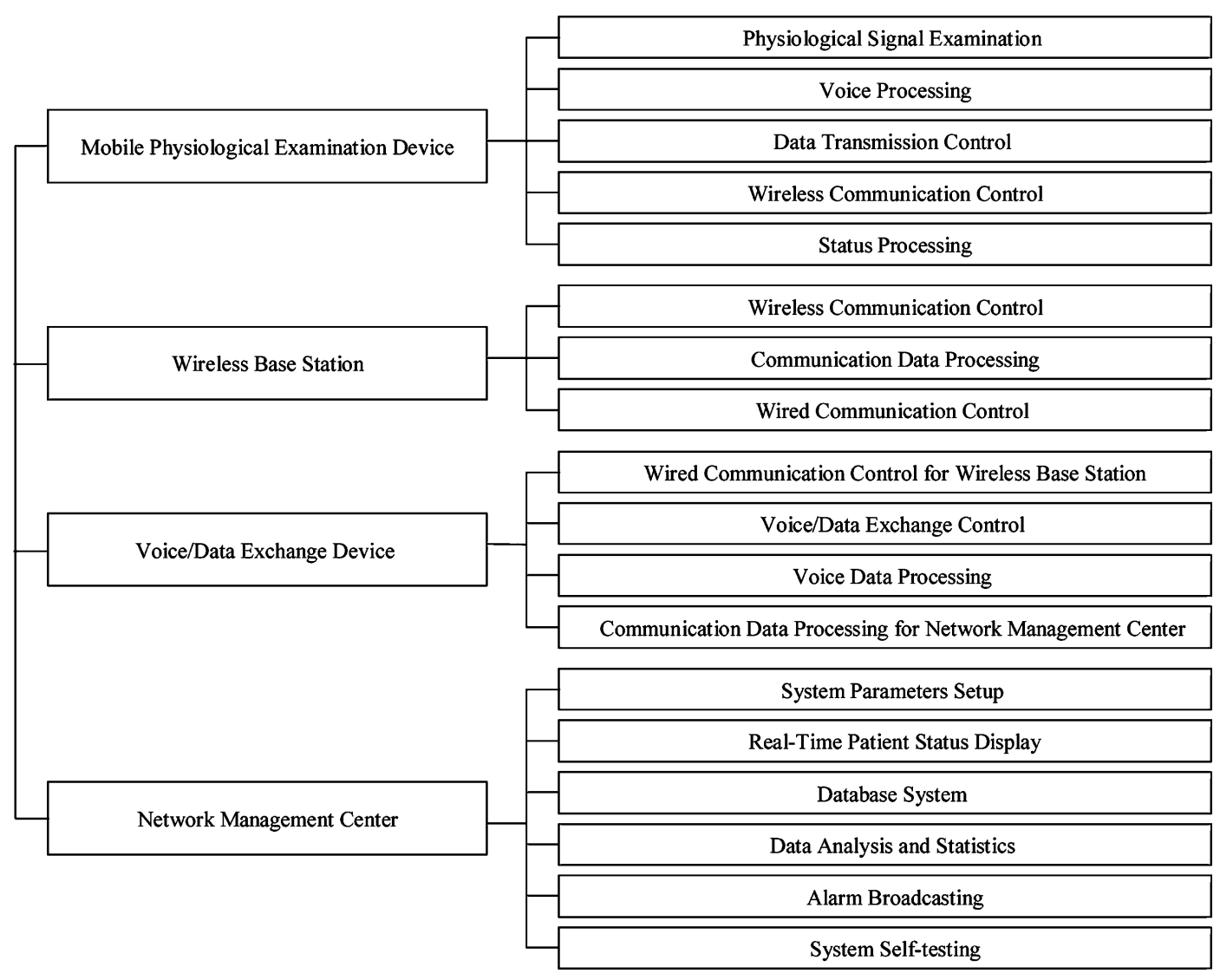

Fig. 7. RTWPMS software architecture.

The application program installed in a wireless base station includes modules for wireless communication control, communication data processing, and wired communication control. These modules take care of data transmission and data conversion between wireless and wired devices and monitor the communication status of all mobile wireless physiological examination devices in the reachable range of a base station. The wireless communication control module provides mobile physiological examination devices to hand-off and roaming across base stations by using a common air interface (CAI) technique. The communication data processing module supports the following functions: Coding process, compression process, and system mutualcontrolled process. The information received from the modules of wireless communication control and wired communication control is decompressed and the coding correctness checked, respectively, by the functions of compression and coding processes. Similarly, the data transmitted must also be processed by this module. The wired communication control module uses the NZR coding and digital phase locked loop (DPLL) to keep synchronous communication with this voice/data exchange device.

\section{B. Embedded System in Voice/Data Exchange Device}

An embedded system has been implemented in the voice/data exchange device. We chose DOS as the operating system and installed our $\mathrm{C}++$ application program on this $\mathrm{PC} / 104 \times 86$ embedded board (ICOP-6070, ICOP Technology Corporation,
Taipei, Taiwan, R.O.C.). The embedded system provides the following advantages: Small, flexible, stable, low cost, and more robust I/O ports, as compared with PC. In the development stage, we used a test card plugged on a PC to develop and test all the programs. When testing was finished, we transplanted the programs to the embedded board. The application program installed in this voice/data exchange device includes the following modules: Wired communication control for wireless base station, voice/data exchange control, voice data processing, and communication data processing for the network management center. These software modules conduct the following operations: Control of wireless base stations, sending and receiving data, voice/data exchange process, voice calling, signal conversion process, and mutual-controlled commands from a network management center.

The most important module is the voice/data exchange control that conducts the voice/data exchange process and builds a bidirectional communication channel according to the given destination information. When receiving a requirement from a wireless base station that demands to establish a data transmission channel, this module will establish the data transmission channel by using a data transmission port. If we want to establish a voice channel, a voice transmission port is used instead. Exchange of the voice/data is processed by the voice/data processing module. When the requirement from a network management center is to establish a voice or data transmission channel, a voice or data transmission channel is built between a client and a 


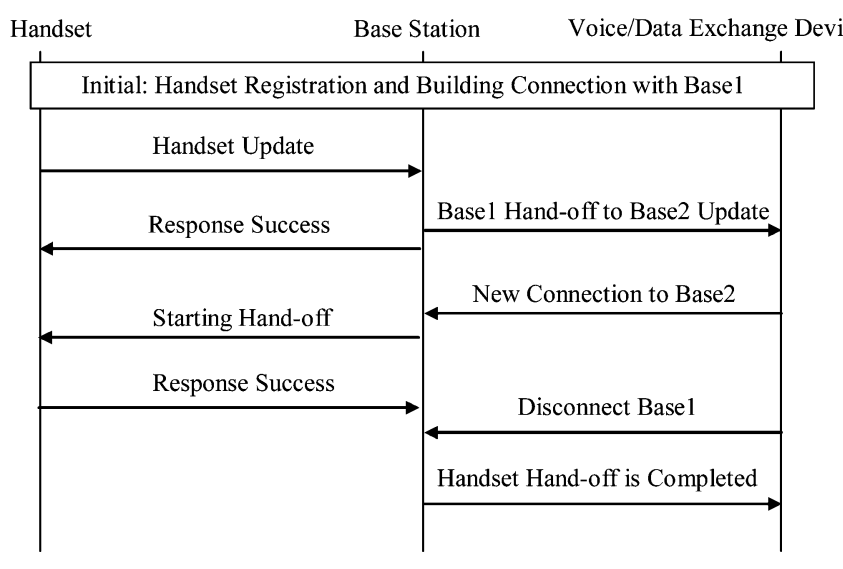

Fig. 8. Procedures of a hand-off.

network management center by the destination information. We also use the voice/data exchange control module to receive the information on the communication quality that is generated by the wired communication control module in a wireless base station. When poor communication quality between a wireless base station and a mobile physiological examination device is found, the voice/data exchange control module compares information of received signal strength from all of the base stations around the mobile physiological examination device and then assigns a new base station to achieve better communication quality. The voice/data exchange control module is responsible for building a communication path for a new base station and for changing the old communication path to this new path. Finally, this software module cancels the old communication path and then finishes a hand off between any two wireless base stations. The exchange procedures are shown in Fig. 8. In such a way, we can overcome communication barriers and reach wireless communication roaming when a patient is moving.

\section{Windows Programs in Network Management Center}

The network management center uses Microsoft Windows 2000 that supports graphic user interface (GUI) and Windows programming capability as its operating platform. The software development platform chosen is Borland $\mathrm{C}++$ Builder, which is an object-oriented software development environment with sufficient reference resources. Borland $\mathrm{C}++$ Builder also provides a powerful database linking function, which allows us to rapidly establish a database for patients. The programmer can easily use the database tools in Borland $\mathrm{C}++$ Builder to access the database content via a network link. For example, Microsoft SQL Server 2000 can be used to access our database.

The application program installed in the network management center includes the following modules: System parameters setup, real-time patient status display, database system, data analysis and statistics, alarm broadcasting, and system selftesting. These application modules are developed under a treelike hierarchical structure, such that all functions in the system can be accessed with a common function interface to reduce the learning curve. With these modules, the medical staff in a network management center can efficiently monitor the status of patients and analyze data stored in the database anytime and anywhere. If patients need help or an accident happens, the staff can give the best response right away.

\section{MEASUREMENTS AND RESUltS}

All of the above discussed hardware devices and software application programs have been integrated into a complete realtime wireless physiological monitoring system. After the independent functions of each device were tested, we began to test the functions of the whole system, including voice transmission, data transmission, programming functions of the network management center, system stability, and reliability between the devices that were mutual controlled. To evaluate the performance of voice and data communication, we used HP 8594E spectrum analyzer to receive radio signals and to analyze spectra. The value of HP 8594E is read and then converted to a received signal strength indicator (RSSI) gradient. RSSI is defined to show whether the received signal strength lies in the golden receiver power rank, such that the transmitter can know the quality of their outgoing data. We used a reference rank of 18 (0.828 V) as the lowest RSSI gradient to test the wireless communication quality and characteristics of the whole system. If the measured value is greater than 18 , it shows that the transmission originates from a base station to which the mobile device has access rights. Our wireless communication devices must guarantee no abnormal effects on both the human body and the medical equipment.

\section{A. Voice Transmission Measurement}

In measuring voice transmission, we generally need to calculate the probability of success in starting a voice transmission between a mobile physiological examination device and a network management center to ensure the voice qualities. After 1000 times of simulated testing, we gathered statistics and found 13 errors, a $1.3 \%$ probability of failure. Most failures happened in the network management center, when software could not link to the mobile physiological examination device in time.

\section{B. Data Transmission Measurement}

Data transmission measurement is focused on testing the wireless communication qualities. A wireless communication channel provides a bidirectional communication with a $32-\mathrm{kb} / \mathrm{s}$ data transfer rate. We disabled voice functions and inputted data into our system with a $30-\mathrm{kb} / \mathrm{s}$ data transfer rate (we reserved $2 \mathrm{~kb} / \mathrm{s}$ for data security and encryption). The maximum bit error rate $(\mathrm{BER})$ obtained is under $6 \times 10^{-6}$. In cases where voice function is enabled, the largest amount of transmitted voice and data is constrained by $32 \mathrm{~kb} / \mathrm{s}$ and the data transfer rate of data transmission is substantially reduced. The data transmission has to use the remaining transmission bandwidth except for status information, mutual-controlled commands, and static sound compression. At this time, the data transfer rate slows down and a large amount of buffer memory is needed. Therefore, we used a $15-\mathrm{kb} / \mathrm{s}$ data transfer rate (we reserved $1 \mathrm{~kb} / \mathrm{s}$ for data security and encryption) for data transmission testing; the maximum BER obtained is under $2 \times 10^{-5}$. 


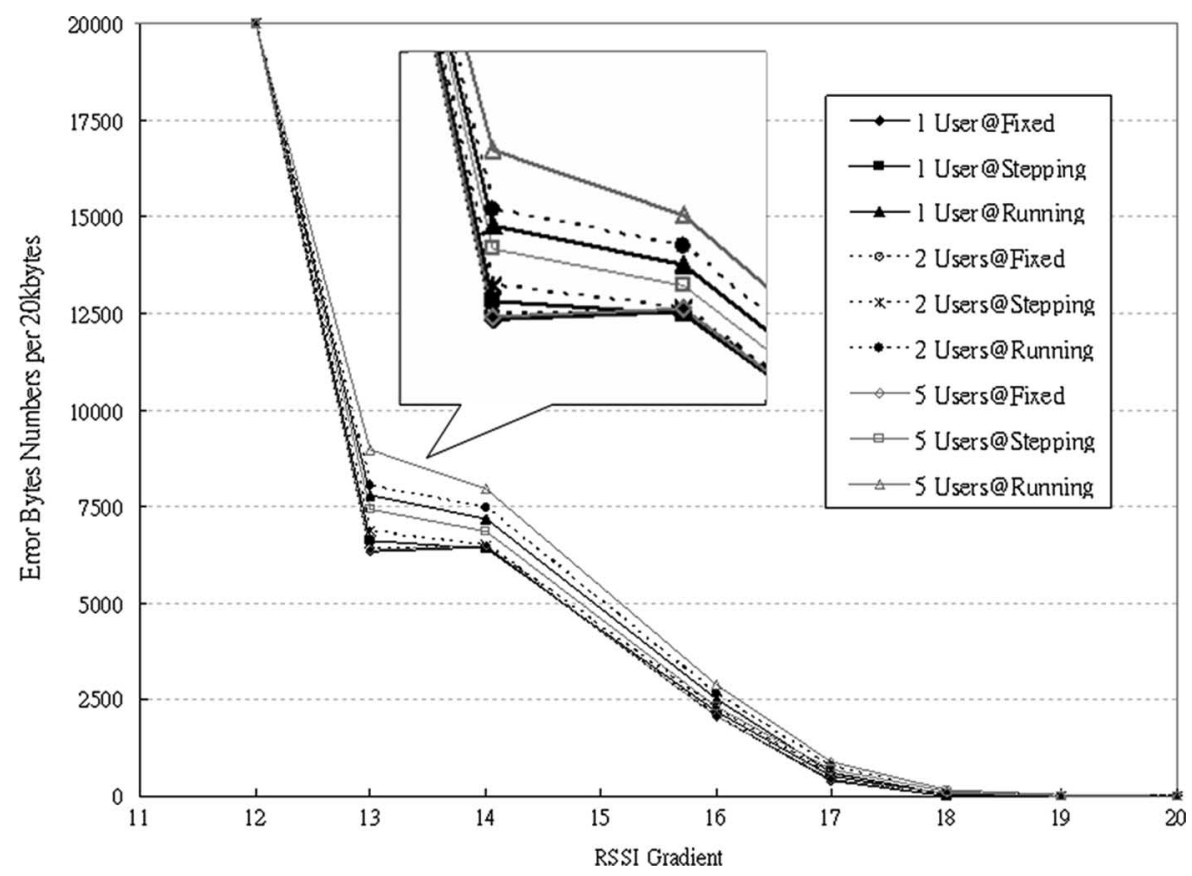

Fig. 9. Error rate trend diagram in a multiuser data transmission testing at fixed, stepping, and running states.

\section{Electromagnetic Interference Detection}

The system is designed to be used in a nursing center and hospital, and thus, we should consider whether it has an impact on medical instruments or human beings. The measurement was finished in an electromagnetic interference semianechoic chamber facility laboratory. We mainly tested our wireless base station and mobile physiological examination device. The measurement referred to the International Special Committee on Radio Interference (CISPR) 11 and 16-1 definitions and limitations as defined by CISPR under the International Electrotechnical Commission (IEC) [18]. When measuring the intensity of electromagnetic radiation at a 3-m distance from the wireless base station, we observed unusual radiation energy from 100 to $230 \mathrm{MHz}$. It might be caused by the switching power circuit in that wireless base station. The intensity of electromagnetic radiation measured is less than $46 \mathrm{~dB} \cdot \mu \mathrm{V}$, which conforms to the definitions and limitations of CISPR 11 electromagnetic interference characteristics. Under the same measuring conditions, the result of measuring mobile physiological examination device shows that the intensity of electromagnetic radiation is less than $40 \mathrm{~dB} \cdot \mu \mathrm{V}$, from 30 to $500 \mathrm{MHz}$ frequency bands, which also conforms to the definitions and limitations of CISPR 11 electromagnetic interference characteristics.

\section{Wireless Communication Detection on the Spot}

The voice and data transmission of this system use wireless communication techniques. Thus, for measurements conducted on the spot, we analyzed distribution of wireless electric field intensity by comparing measurement data with the results from a better communication environment in the laboratory to ensure that our system can operate well in the nursing centers. Measurements conducted on the spot are performed inside and outside nursing centers or hospitals. The indoor measurements include electric field intensity of external wireless communication, power line of internal equipments, wired signal transmission line, and electronic medical instruments. The outdoor measurements include electric field intensity of external wireless communication and power line. We used a spectrum analyzer to measure the wireless electric field intensity and the distribution status of electric field in frequency domain to analyze the distribution of electric field. After indoor measurements were taken, we found the electromagnetic interference strength from outdoor was less than $-55 \mathrm{~dB} \cdot \mathrm{m}$ and distributed over other frequency bands that were different from our system. Our system had little interference in an indoor environment. In the outdoor environment, we found that many electromagnetic interference sources, such as cellular phone base stations, influenced our system. We found an electromagnetic interference of up to $-12 \mathrm{~dB} \cdot \mathrm{m}$ near a global system for mobile (GSM) base station. To solve the electromagnetic interference problem, we should keep away from those interference sources or add more base stations into our system.

Fig. 9 shows the error rate in a multiuser data transmission testing at fixed, stepping, and running states. We found that under an excellent communication environment, the communication results are the same and there are no transmission errors (RSSI $>20)$. When the patients are motionless, multiuser error rate is greater than a single-user error rate. The reason might be that wireless communication signals from our system interfere with each other to cause a signal-to-noise ratio (SNR) decrease when the RSSI is low, omitting the noise interference of environment. We analyzed the measured results with the same number of users, respectively, in the states of walking (about $1.2 \mathrm{~m} / \mathrm{s}$ ) and running (about $4 \mathrm{~m} / \mathrm{s}$ ). Fig. 9 shows that the moving speed of patients has more effect than the user number. When a patient's 
TABLE II

RTWPMS SPECIFICATIONS

\begin{tabular}{|c|c|}
\hline \multicolumn{2}{|c|}{ Communication Specifications } \\
\hline Frequency & $864 \mathrm{MHz}$ \\
\hline Channels & 40 \\
\hline Data Rate & $32 \mathrm{~kb} / \mathrm{s}$ \\
\hline Power & $<10 \mathrm{~mW}$ \\
\hline \multicolumn{2}{|c|}{ Wireless Communication Distance (RSSI $=18$ ) } \\
\hline Indoor & Laboratory: $80 \mathrm{~m}$, Real Test: $40 \sim 50 \mathrm{~m}$ \\
\hline Outdoor & Laboratory: $200 \mathrm{~m}$, Real Test: 160 200m \\
\hline \multicolumn{2}{|l|}{ BER of Data Transmission } \\
\hline Data Only & $<6 \times 10^{-6} @ 30 \mathrm{~kb} / \mathrm{s}$ \\
\hline Data + Voice & $<2 \times 10^{-5} @ 15 \mathrm{~kb} / \mathrm{s}$ \\
\hline \multicolumn{2}{|c|}{ Handset Power Consumption } \\
\hline Idle & $<1.5 \mathrm{~mA} @ 7.2 \mathrm{~V}, 1850 \mathrm{mAH}$ Lithium-Ion Battery \\
\hline Handshake & $<14 \mathrm{~mA} @ 7.2 \mathrm{~V}, 1850 \mathrm{mAH}$ Lithium-Ion Battery \\
\hline Voice Transmission & $<50 \mathrm{~mA} @ 7.2 \mathrm{~V}, 1850 \mathrm{mAH}$ Lithium-Ion Battery \\
\hline Data Transmission & $<70 \mathrm{~mA} @ 7.2 \mathrm{~V}, 1850 \mathrm{mAH}$ Lithium-Ion Battery \\
\hline Voice+Data Transmission & $<80 \mathrm{~mA} @ 7.2 \mathrm{~V}, 1850 \mathrm{mAH}$ Lithium-Ion Battery \\
\hline \multicolumn{2}{|c|}{ Built-in Medical Examination Modules } \\
\hline \multirow[t]{5}{*}{ Blood Pressure } & Refer to ANSI/AAMI SP10-92 \\
\hline & Max. $\pm 5 \%$ Error@ Standard Pressure Module in Laboratory \\
\hline & Max.土9mmHg Error@ Medical Examination Module Used in Hospital. \\
\hline & Max. $\pm 17 \mathrm{mmHg}$ Error@ BP-Systolic Compared with Equipment in Hospital \\
\hline & Max. $\pm 9 \mathrm{mmHg}$ Error@ BP-Diastolic Compared with Equipment in Hospital \\
\hline \multirow[t]{4}{*}{ Body Temperature } & Refer to ASTM E1112-86 \\
\hline & Max. $\pm 0.1^{\circ} \mathrm{C}$ Error @ Standard Temperature Module in Laboratory. \\
\hline & Max. $\pm 0.2^{\circ} \mathrm{C}$ Error@ Medical Examination Module Used in Hospital. \\
\hline & Max. $\pm 0.4^{\circ} \mathrm{C}$ Error Compared with Equipment in Hospital \\
\hline \multirow[t]{3}{*}{ Heart Rate } & Max.土8 Error@ @ Blood Pressure Module Used in Hospital \\
\hline & Max.土16 Error@ Pressure Module Used in Hospital \\
\hline & Max. \pm 3 Error Compared with Equipment in Hospital \\
\hline
\end{tabular}

moving distance increases, the error rate for the case of patients in the state of running is greater than the case in walking, and the error rate in the case of patients in the state of walking is greater than the case of patients at rest. That is, we have a greater noise variation in an environment as RSSI decreases. Also, the received signals of the mobile physiological examination devices will have larger variations due to variations of response time in autogain control (AGC).

\section{E. Results}

The system has been tested on the spot in nursing centers and hospitals, and it functions normally. The study was conducted on 17 healthy volunteers; any volunteering healthy subject aged from 23 to 75 . Fig. 10 presents three vital signals of a walking 68 -year-old volunteer. It clearly shows the value and trend of body temperature, systolic BP, diastolic BP, and HR that were collected from the mobile physiological examination device. In this case, the body temperature was measured once every $5 \mathrm{~s}$, BP was measured once every hour, and HR was measured once every $10 \mathrm{~s}$. From the results shown above, a summary of the system characteristics is provided in Table II, showing that our RTWPMS can successfully transmit patient physiological parameters as expected. Compared with the data measured by medical instruments in nursing centers and hospitals, our physiological status monitoring system has reached the application level. In studying the interference with other medical instruments, the

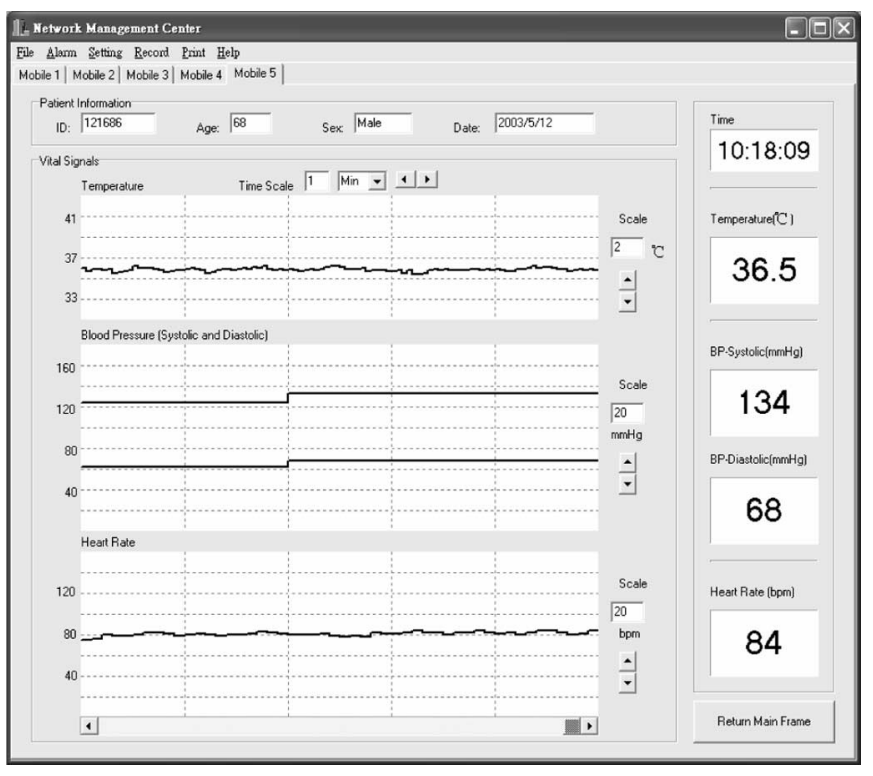

Fig. 10. Snapshot of the Windows programs network management center that displays three vital signals: body temperature, BP, and HR.

results showed that our proposed system would not influence most medical instruments at a short distance. Transmitting data and voice together could be achieved under a $2 \times 10^{-5}$ BER. Our supported voice calling function provides the nursing staff 
and patients with a mobile voice conversation capability, which makes the system more practical and valuable.

Regarding the human-machine interface, the system provides functions such as real-time information and record searching, windows operation, GUI, and external network communication. The medical staff helped us to modify the GUI into a form suitable for usage. The application of our system in nursing centers and hospitals can replace a large amount of their staffing hours and provide a more efficient medical nursing service.

\section{CONCLUSION}

In this paper, we presented the design and implementation of an RTWPMS that consists of mobile physiological examination device(s), wireless base station(s), a voice/data exchange device, and a network management center. The modular approach applied in hardware and software design enables the RTWPMS to be configurable for different application scenarios. For example, the mobile physiological examination device supports four RS-232 ports, which can be used to connect several optional medical examination modules in addition to the built-in one. Moreover, the wireless communication module in this examination device can be replaced by other modules such as a wireless local area network (WLAN) module. In addition, this system is feasible for further extension. Network communication, GPS, and emergency calling all can easily be integrated within this system. Therefore, our system can adapt to the requirements of many different environments and can be upgraded by using new technologies.

The system has been tested on the spot at nursing centers and hospitals. The wireless communication distance can reach $50 \mathrm{~m}$ when the system is used indoors or $150 \mathrm{~m}$ when used outdoors. It provides the functions of real-time physiological monitoring, portability, bidirectional voice, and data transmission. The nursing staff equipped with the functions of this system can monitor online the real-time status and activities of patients and can supply better service.

The proposed RTWPMS has advantages in that it is bidirectional, communicates without charge, and operates independently. Thus, it is especially suitable to be used in self-contained and small places such as nursing centers that require many staffing hours to look after their patients. Power consumption of our proposed mobile physiological examination device is far less than $10 \mathrm{~mW}$; it can, thus, provide a safer and longer monitoring. Since the system is operating independently, it can increase stability and reduce communication expense. These new features not only make the system convenient to use, but also play an important role in patient monitoring and treatment. The RTWPMS really provides a better solution for nursing centers, in comparison with other productions.

\section{ACKNOWLEDGMENT}

The authors would like to thank NTU Hospital, En Chu Kong Hospital, and Taipei City Psychiatric Center in Taipei, Taiwan, R.O.C., for their supporting clinical examinations.

\section{REFERENCES}

[1] K. Doughty, K. Cameron, and P. Garner, "Three generations of telecare of elderly," J. Telmed. Telecare, vol. 2, no. 2, pp. 71-80, 1996.

[2] P. Johnson and D. C. Andrews, "Remote continuous physiological monitoring in the home," J. Telmed. Telecare, vol. 2, no. 2, pp. 107-113, 1996.

[3] M. J. Rodriguez, M. T. Arredondo, F. del Pozo, E. J. Gomez, A. Martinez, and A. Dopico, "A home telecare management system," J. Telmed. Telecare, vol. 1, no. 2, pp. 86-94, 1995.

[4] G. Williams, P. J. King, A. M. Capper, and K. Doughty, "The electronic doctor (TED)-A home telecare system," in Proc. 18th IEEE Annu. EMBS Int. Conf., Amsterdam, The Netherlands, Oct. 31-Nov. 3, 1996, vol. 1, pp. 53-54.

[5] J. Bai et al., "The design and preliminary evaluation of a home electrocardiography and blood pressure monitoring network," J. Telmed. Telecare, vol. 2, no. 2, pp. 100-106, 1996.

[6] P. Varady, Z. Benyo, and B. Benyo, "An open architecture patient monitoring system using standard technologies," IEEE Trans. Inf. Technol. Biomed., vol. 6, no. 1, pp. 95-98, Mar. 2002.

[7] L. Li, Z. Wang, and K. Zhang, "CWANT: A telemedical systems in China," in Proc. 1st Joint BMES/EMBS Conf. Serving Humanity, Advancing Technol., Atlanta, GA, Oct. 13-16, 1999, vol. 2, p. 706.

[8] M. Takizawa, S. Sone, K. Hanamura, and K. Asakura, "Telemedicine system using computed tomography van of high-speed telecommunication vehicle," IEEE Trans. Inf. Technol. Biomed., vol. 5, no. 1, pp. 2-9, Mar. 2001.

[9] R. H. Istepanian, M. Brien, P. Smith, M. Hays, and R. Naimimohasses, "Modelling of photoplethysmography mobile telemedicine system," in Proc. 19th IEEE/EMBS Int. Conf., Chicago, IL, Oct. 30-Nov. 2,1997, vol. 3, pp. 987-990.

[10] S. Pavlopoulos, E. Kyriacou, A. Berler, S. Dembeyiotis, and D. Koutsouris, "A novel emergency telemedicine system based on wireless communication technology_AMBULANCE," IEEE Trans. Inf. Technol. Biomed., vol. 2, no. 4, pp. 261-267, Dec. 1998.

[11] B. Woodward, R. S. H. Istepanian, and C. I. Richards, "Design of a telemedicine system using a mobile telephone," IEEE Trans. Inf. Technol. Biomed., vol. 5, no. 1, pp. 13-15, Mar. 2001.

[12] K. Huang and Y.-T. Zhang, "Implementation of a wap-based telemedicine system for patient monitoring," IEEE Trans. Inf. Technol. Biomed., vol. 7, no. 2, pp. 101-107, Mar. 2003.

[13] R. S. H. Istepanian, "Modelling of GSM-based mobile telemedical system," in Proc. 20th IEEE/EMBS Conf., Hong Kong, China, Oct. 29-Nov. 1, 1998, vol. 3, pp. 1166-1169.

[14] Y. Liu, "The design and implementation of a virtual medical centre for patient home care," in Proc. 20th IEEE/EMBS Conf., Hong Kong, China, Oct. 29-Nov. 1,1998, vol. 3, pp. 1163-1165.

[15] E. Jovanov, J. Price, D. Raskovic, K. Kavi, T. Martin, and R. Adhami, "Wireless personal area networks in telemedical environment," in Proc. 3rd IEEE/EMBS Inf. Technol. Appl. Biomed. Workshop Int. Telemed. Inf. Soc., Arlington, VA, Nov. 9-10, 2000, pp. 22-27.

[16] G. C. Crumley, N. E. Evans, W. G. Scanlon, J. Brian, and T. G. Trouton, "The design and performance of a $2.5-\mathrm{GHz}$ telecommand link for wireless biomedical monitoring," IEEE Trans. Inf. Technol. Biomed., vol. 4, no. 4, pp. 285-291, Dec. 2000

[17] A. I. Hernandez, F. Mora, G. Villegas, G. Passariello, and G. Carrault, "Real-time ECG transmission via Internet for nonclinical applications," IEEE Trans. Inf. Technol. Biomed., vol. 5, no. 3, pp. 253-257, Sep. 2001.

[18] International Special Committee on Radio Interference, Limits and methods of measurement of electromagnetic disturbance characteristics of industrial, scientific and medical ISM radio-frequency equipment CISPR 11/EN 55011/CNS13803, CISPR 11/IEC 60601-1-2 CNS 13803, 13804/FCC Part 18, CISPR 16-1.

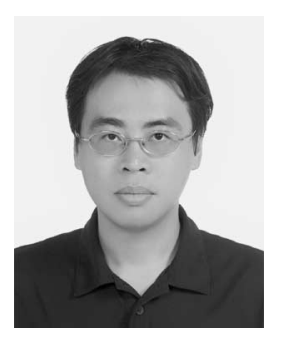

Bor-Shing Lin was born in Changhua, Taiwan, R.O.C., in 1973. He received the B.S. degree from National Cheng Kung University, Tainan, Taiwan, R.O.C., in 1997, and the M.S. degree from National Taiwan University, Taipei, Taiwan, R.O.C., in 1999, both in electrical engineering. He is currently working toward the Ph.D. degree in electrical engineering at National Taiwan University.

His research interests include EEG systems, medical informatics, wireless communication systems, embedded systems, and RF IC design. 


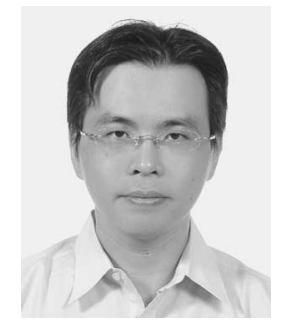

Bor-Shyh Lin (S'02-M'03) was born in Changhua, Taiwan, R.O.C., in 1975. He received the B.S. degree from National Chiao Tung University, Hsinchu, Taiwan, R.O.C., in 1997, and the M.S. degree from National Taiwan University, Taipei, Taiwan, R.O.C., in 1999, both in electrical engineering. He is currently working toward the Ph.D. degree in electrical engineering National Taiwan University.

His research interests include EEG systems, medical informatics, and biomedical signal processing.

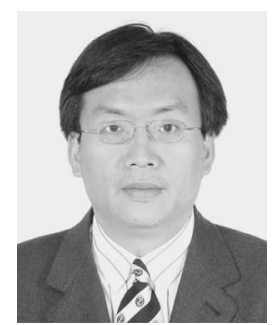

Nai-Kuan Chou received the M.D. degree from the Department of Medicine, Medical College, National Taiwan University, Taipei, Taiwan, R.O.C., in 1988, and the Ph.D. degree in electrical engineering from National Taiwan University, in 2001.

He joined California Pacific Medical Center, San Francisco, in 1993 and again in 1996. Currently, he is a Clinical Assistant Professor of Surgery at National Taiwan University Hospital, Taipei. His research interests include cardiovascular surgery, ventricular assist devices extracorporeal membrane oxygenation, heart transplantation, critical medicine, and medical informatics.

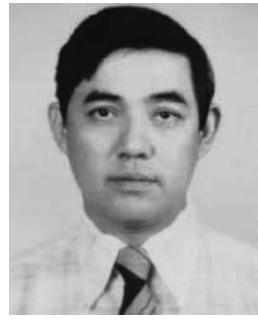

and design

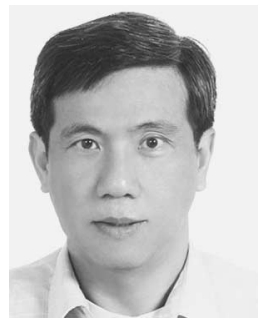

Fok-Ching Chong received the B.S. degree in electrical engineering from National Taiwan University, Taipei, Taiwan, R.O.C., in 1971.

Since 1971, he has been with the Department of Electrical Engineering (EE), National Taiwan University. During 1976-1989, he was the Head of the Operation Division, University Computer Center. $\mathrm{He}$ is the Group Leader for the Medical Engineering Division of the EE Department, National/Taiwan University. His research interests include biomedical signal processing, simulations, and system analysis

Sao-Jie Chen received the B.S. and M.S. degrees from National Taiwan University, Taipei, Taiwan, R.O.C., in 1977 and 1982, respectively, and the Ph.D. degree from Southern Methodist University, University Park, TX, in 1988, all in electrical engineering.

Since 1982, he has been a member of the faculty in the Department of Electrical Engineering, National Taiwan University, where he is currently a Full Professor. He was a Visiting Professor at the University of California, San Diego (1999); an Academic Visitor at the IBM Thomas J. Watson Research Center, Yorktown Heights, NY (2003); and a Visiting Professor at the University of Wisconsin, Madison (2004 and 2005). His current research interests include VLSI physical design, wireless LAN and Bluetooth IC design, and SOC hardware/software codesign.

Dr. Chen is a member of the Chinese Institute of Engineers, the Chinese Institute of Electrical Engineering, and the Association for Computing Machinery, and a Senior Member of the IEEE Circuits and Systems and IEEE Computer Societies. 